Ann. Zootech., I969, 18 (I), 55-64.

\title{
INFLUENCE D'UN APPORT CROISSANT D'ORGE SUR LE COMPORTEMENT ALIMENTAIRE DE LA VACHE LAITIÈRE ET LA DIGESTION
}

\author{
B. REMOND \\ avec la collaboration technique de Jeanne TLíchet et A. Ot.titer \\ Station de Recherches sur l'Élevage des Ruminants, \\ Centre de Recherches zootechniques et vétérinaires sur les Ruminants, \\ 63 - Theix, près Clermont-Ferrand \\ Institut national de la Recherche agronomique
}

SOMMAIRE

Trois vaches taries portant une fistule du rumen ont reçu du foin de luzerne normal ad libitum et une quantité croissante d'orge broyée. On a étudié les modifications de leur comportement alimentaire (tabl. I) et de leur digestion.

La quantité de matière sèche ingérée augmente jusqu'à ce que l'orge représente de 35 à 45 p. roo de la ration (fig. I). Cette augmentation s'accompagne d'un accroissement de la vitesse de transit digestif de l'orge et du foin (fig. 2), des quantités de contenus digestifs du rumen et de la digestibilité de la ration (tabl. 2); la vitesse des fermentations dans le rumen (fig. 3) augmente également, bien que l'activité cellulolytique du jus de rumen diminue.

Au-delà de 35 à 45 p. Ioo d'orge, la quantité de matière sèche ingérée diminue; la vitesse des fermentations dans le rumen n'augmente plus ; les vitesses de transit de l'orge et du foin ont tendance à diminuer ; la quantité de contenu digestif du rumen décroît. Les informations qui limitent alors l'ingestion ne doivent donc plus provenir du rumen; elles proviendraient des compartiments digestifs postérieurs on seraient d'origine métabolique mais elles pourraient aussi résulter d'une diminution de l'appétence de la Vache car la vitesse d'ingestion diminue ì partir de $55 \mathrm{p}$. Ioo d'orge (tabl. т).

\section{INTRODUCTION}

L'intensification de la production laitière et la mécanisation de l'alimentation conduisent à accroitre la quantité d'aliments concentrés distibués aux vaches. Ces aliments modifient le niveau d'ingestion du fourrage de façon différente, en particulier selon la quantité qu'on en distribue, et la qualité et la nature du fourrage. Il en résulte une variation du niveau d'ingestion de la ration totale. Ainsi, en faisant varier le rapport fourrage/aliments concentrés, KESLER et SPAHR (Ig63) constatent qu'il 
existe une zone de valeurs pour lesquelles la quantité d'énergie ingérée est maximale. De plus, ces régimes peuvent s'avérer défavorables à la production laitière en diminuant le taux butyreux du lait (Rook, I96I).

L'objet de cette étude préliminaire a été double ; d'une part enregistrer les variations du comportement alimentaire de la Vache lorsqu'on la fait passer d'un régime de foin seul à un régime comportant une proportion croissante d'orge; d'autre part, étudier l'influence de la quantité d'orge sur la digestion de la ration, principalement au niveau du rumen.

\section{MATÉRIEL ET MÉTHODES}

Nous avons expérimenté sur 3 vaches adultes taries, 2 Pie Rouge (449 et 450 ) et une Pic Noire (535), qui portaient une large fistule du rumen. L'essai a commencé le 3 janvier 1966. Pendant les 3 premières semaines, les animaux ont reçu le foin de luzerne à volonté et $\mathrm{x}$ kg d'orge ; ensuite le foin restant distribué à volonté, on a augmenté chaque jour la quantité d'orge offerte d'abord de $200 \mathrm{~g}$, puis de $250 \mathrm{~g}$ à partir du 3 mars pour les vaches 450 et 535 , mais les accroissements de quant ités ingérées furent inférieurs car ces vaches firent alors des refus.

Les aliments utilisés étaient une orge finement broyée additionnée de 3 p. roo de compléments minéraux et ayant une teneur en matières azotées totales de 12,I p. I00 (par rapport à la MS (1)) et un foin de luzerne long, récolté au stade "bourgeons floraux " et dont la teneur en cendres était de I I, 4 P. 100, en matières azotées totales de 14,2 P. Ioo et en cellulose brute de 30,0 p. I0o. La digestibilité de la matière organique de ce foin mesurée sur 4 moutons était de $57, \mathrm{r} \mathrm{p}$. roo. L'orge était distribuée à $5 \mathrm{~h} 30$ et $\mathrm{I} 7 \mathrm{~h} 30$, et le foin à 6 heures et 18 heures, après enlèvement de's refus (environ ro $\mathrm{p}$. roo pour le foin) de la distribution précédente. Les animaux avaient en permanence les aliments à leur disposition.

La Vache 449 a été retirée de l'expérience le 20 février puis abattue à la suite de l'obturation de sa sonde urétrale, et remplacée jusqu'au 30 avril par la Vache 5.35 dont l'alimentation ćtait identique. La Vache 450 a été éliminée le 30 mars à la suite d'une indigestion.

Nous avons effectué, au cours de périodes expérimentales successives de 2 semaines, les mesures suivantes : comportement alimentaire, digestibilité de la ration (pendant 7 jours), activité cellulolytique du jus de rumen (avec $2 \mathrm{~g}$ de papier-filtre pendant 48 heures), vitesse de fermentation du contenu du rumen, poids de contenu de rumen, pH du jus de rumen, dans les conditions exposées par Journet et DEMARQUILIY (1967).

Du jus de rumen a été prélevé de façon continue pendant 24 heures avec une pompe Sigmamotor. Le prélevement a été répété 2 jours consécutifs (GACSSEREs, 1965). La concentration en A(iV ( $\left.{ }^{2}\right)$ a été mesurée par la méthode de Vandennieuvis (1965) et leur séparation a été effectuée sur une colonne de silicone avec un chromatographe en phase gazeuse muni d'un détecteur à ionisation de flamme ${ }^{(3)}$.

\section{RÉSULTATS}

\section{Comportement alimentaire (fig. I, tabl. I)}

Lorsque la quantité d'orge distribuée s'accroît, la quantité de foin ingérée ne diminue pas immédiatement (Vache 535) ou diminue moins rapidement que l'apport d'orge n'augmente (respectivement de $0,69 \mathrm{~kg}$ et 0,48 $\mathrm{kg}$ par $\mathrm{kg}$ d'orge supplémentaire ingérée pour les vaches 449 et 450 ). Il en résulte que la quantité totale de MS ingérée augmente jusqu'à un maximum ; elle est alors plus élevée qu'initialement, respecti-

(1) MS : matière sìche.

(2) AGV : acides gras volatils.

(3) Le protocole de la méthode sera publié ultérieurement. 
APPORT CROISSANT D'ORGE CHEZ LA VACHE LAITIÈRE

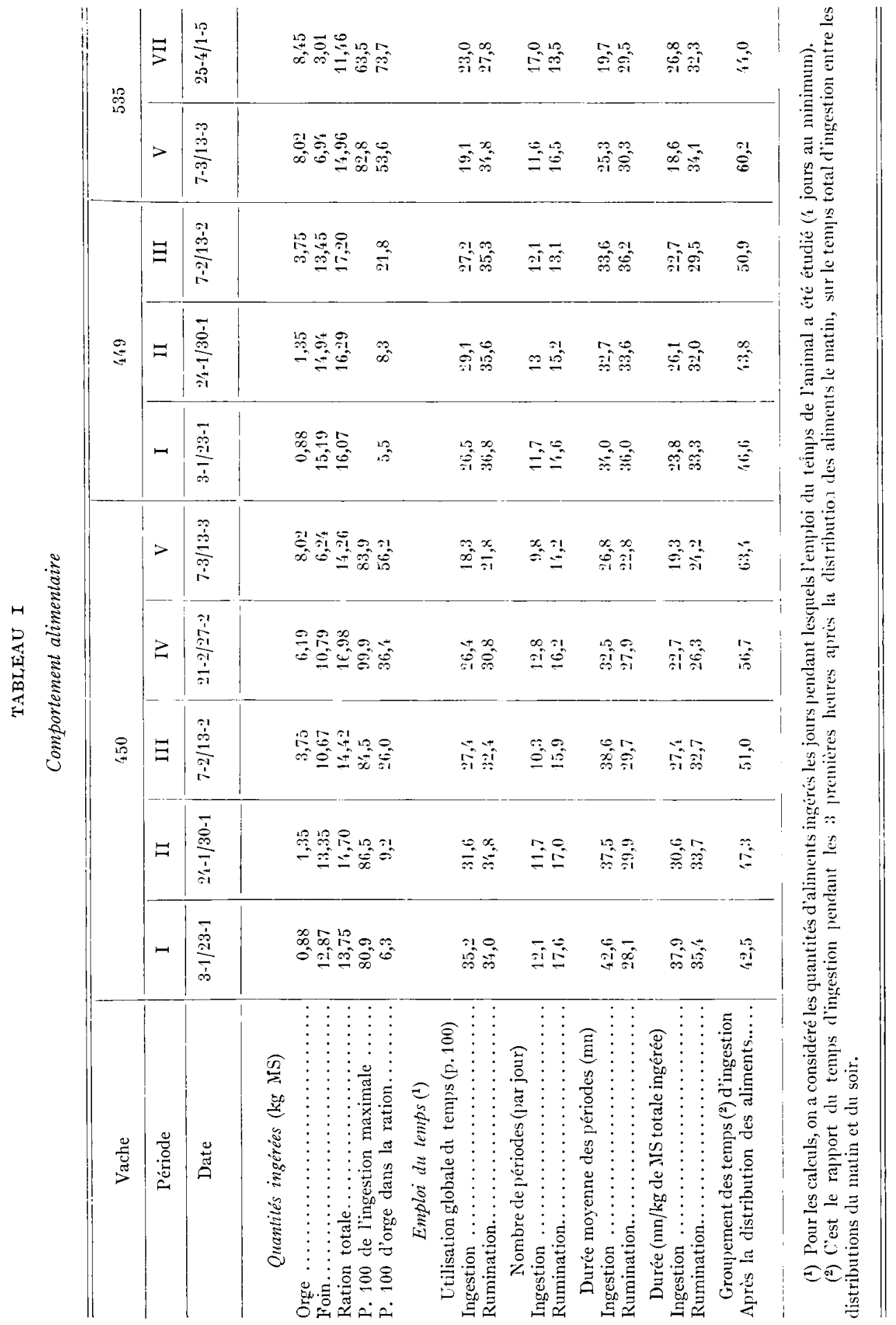


vement de $3,25 \mathrm{~kg}$ (23 p. IOO) et $6,10 \mathrm{~kg}$ (5I p. IOO) pour les deux vaches. Puis la quantité de foin ingérée commence à diminuer (Vache 535) ou diminue plus vite (Vache 450), et la quantité totale de MS ingérée décroît.

Les vaches $45^{\circ}$ et 535 ont commencé à ne pas consommer la totalité de l'orge lorsqu'elles en ont reçu environ $8 \mathrm{~kg}$, soit approximativement $55 \mathrm{p}$. Ioo de la ration. A partir de cette proportion, l'ingestion fut moins régulière.

L'emploi du temps a été étudié par la méthode d'enregistrement graphique de Ruckebusch (1963) sur les vaches 449 (3 périodes entre 5 p. Ioo et 22 p. Ioo d'orge dans la ration), $45^{\circ}$ ( 5 périodes entre $6 \mathrm{p}$. Ioo et $56 \mathrm{p}$. IO0) et 535 (2 périodes pour 54 et 74 p. IOo). Les chiffres que nous donnons sont la moyenne d'au moins quatre jours d'enregistrement.
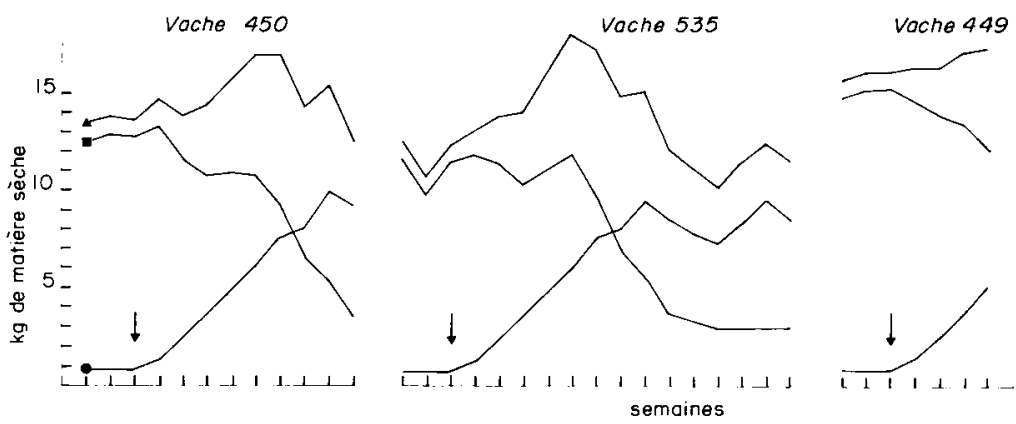

FIG. I. - Quanlités ingérées

- Ration totale

- Foin

- Orge

$\downarrow$ Début de la distribution d'orge

Entre les périodes I et 5 (6 à 56 p. I oo d'orge dans la ration), le temps d'ingestion de la Vache 450 (exprimé en pourcentage du temps total) diminue environ de moitié, le temps de rumination de $35 \mathrm{p}$. Ioo et le nombre journalier de périodes d'ingestion et de rumination de I9 p. Ioo (tabl. I). Il en est de même de la durée des périodes d'ingestion ( $37 \mathrm{p}$. I0o) et de rumination ( 8 p. IOO). La diminution du temps d'ingestion est donc principalement due à la diminution de la durée des périodes d'ingestion tandis que la diminution du temps de rumination est due également à la diminution du nombre de périodes. En conséquence, les temps d'ingestion et de rumination par $\mathrm{kg}$ de MS ont respectivement diminué de $47 \mathrm{p}$. Ioo et $3 \mathrm{I}$ p. Ioo. De plus, une proportion plus importante du temps d'ingestion a été passée au cours des trois heures qui suivaient la distribution des aliments du matin.

Les données enregistrées sur les vaches 449 et 535 sont trop limitées pour que l'on puisse dégager une évolution. Chez la Vache 449 on observe peu de variations de l'emploi du temps entre 5 et 22 p. Ioo d'orge dans la ration. Cependant, lorsque la Vache 535 passe d'une ration où elle choisit librement ses aliments (période 5) à une ration oì la quantité de foin distribué est limitée à $3 \mathrm{~kg}$ par jour (période 7), le temps d'ingestion par $\mathrm{kg}$ de MS augmente de 44 p. Ioo alors que celui de rumination a tendance à diminuer.

Ces résultats, principalement ceux qui concernent les vaches 450 et 535, suggèrent que, dans nos conditions expérimentales, la vitesse d'ingestion est d'autant plus 


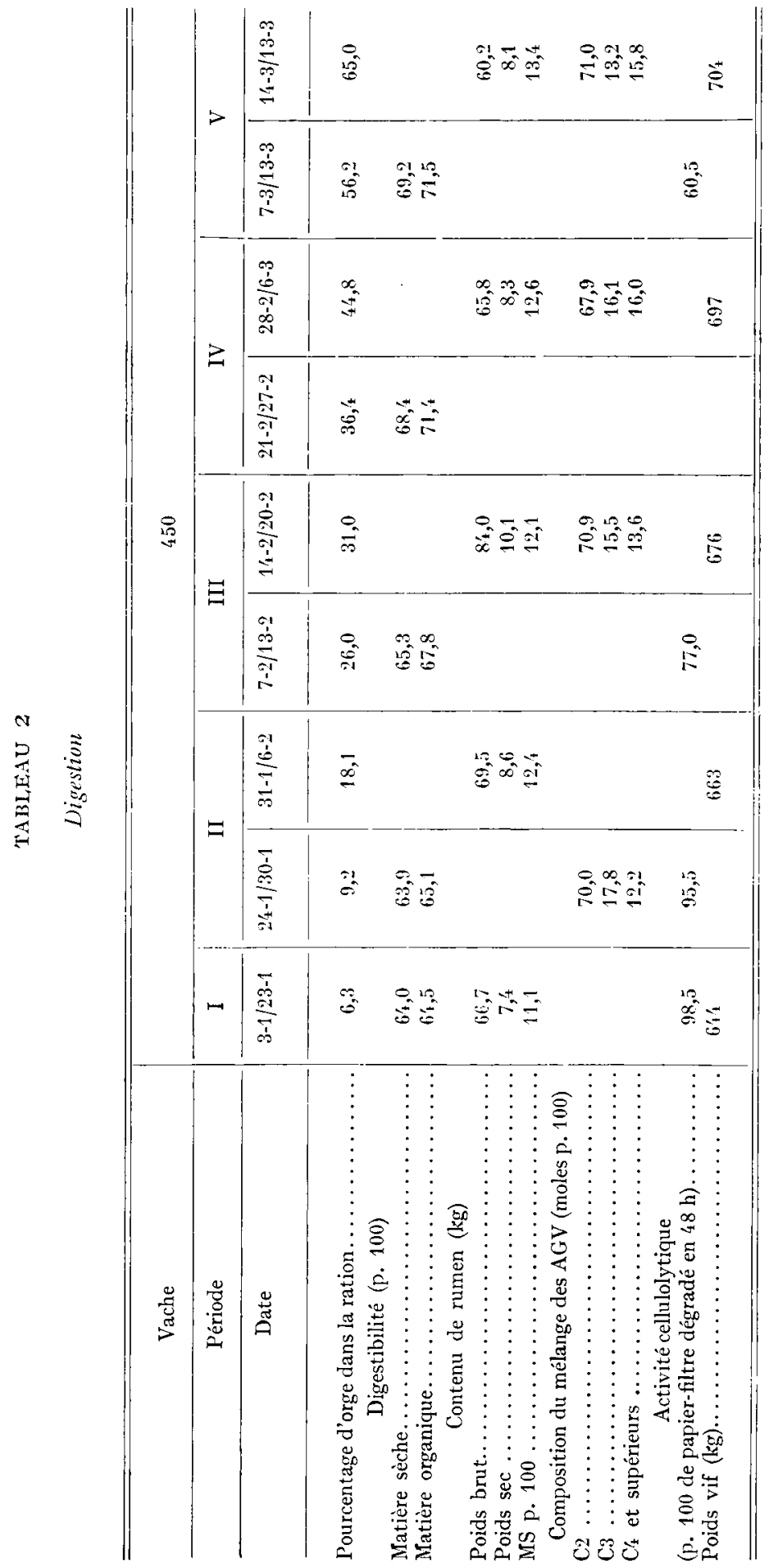


B. RÉMOND

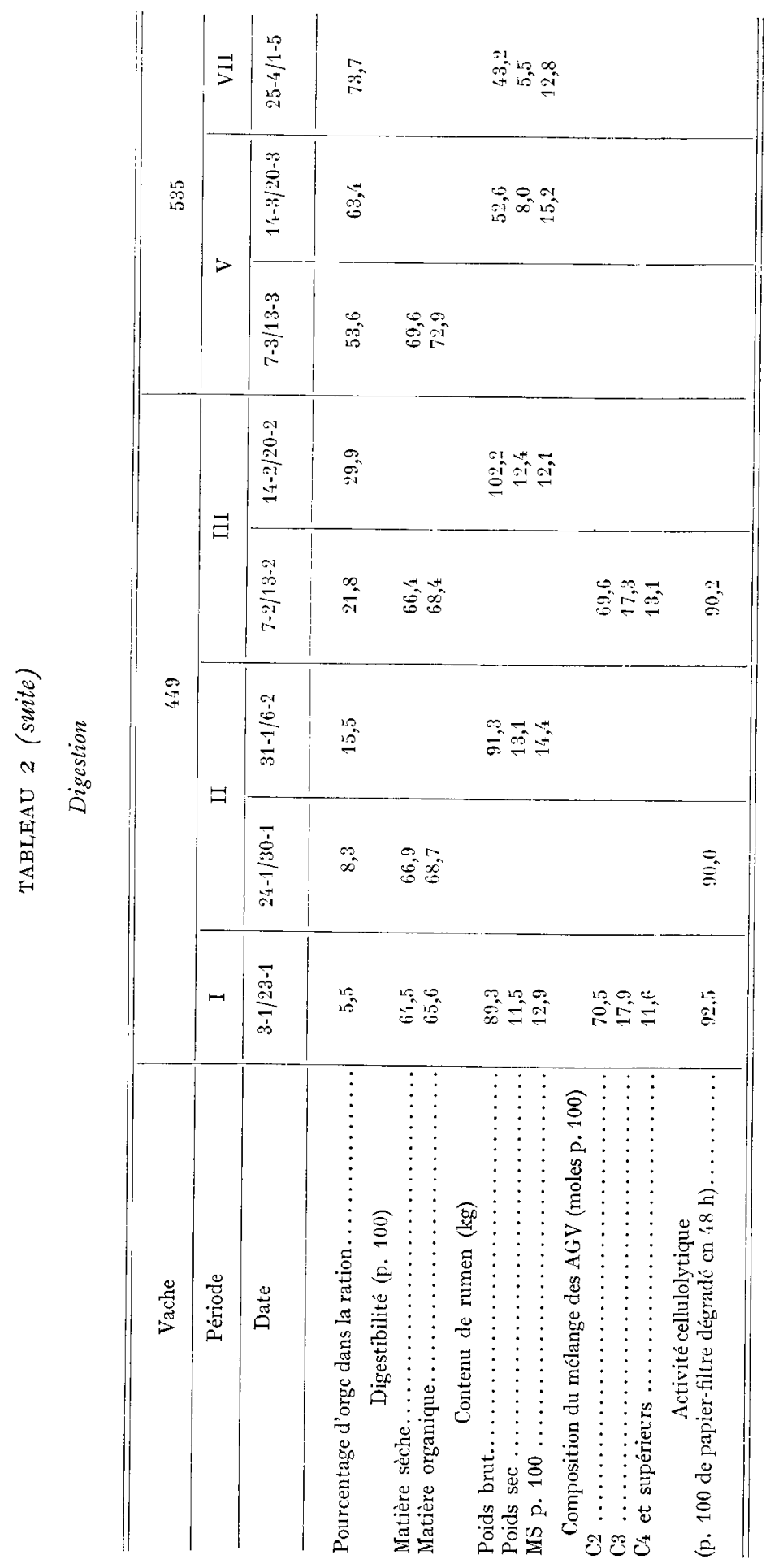


grande que l'animal est plus libre de choisir ses aliments. Ils semblent montrer aussi que la rumination dépend étroitement de l'état physique de la ration alors que 1'ingestion dépend aussi des " goûts " de l'animal.

\section{Digestion}

Les résultats sont rapportés au tableau 2. Quand le pourcentage d'orge dans la ration augmente, la digestibilité de la ration croît d'abord fortement puis faiblement. La digestibilité du foin calculée en admettant une digestibilité constante de 88 pour celle de l'orge est, pour les 5 périodes de mesures effectuées sur la Vache $45^{\circ}$, de $62,9-60,7-6 \mathrm{I}, 8$ et 50,3. La forte diminution ainsi calculée entre les périodes 4 et 5 peut également provenir d'une diminution de digestibilité de l'orge. L'augmentation de digestibilité de la ration et les quantités ingérées ont provoqué une augmentation de poids vif des animaux. Chez la Vache $45^{\circ}$, elle a été de $60 \mathrm{~kg}$ en 8 semaines, soit I o7o grammes par jour.

Les temps de séjour de l'indigestible du foin et de l'orge dans le tube digestif sont rapportés à la figure 2. L'ensemble des résultats obtenus sur les trois animaux montre que le temps de séjour des deux aliments passe par un minimum pendant que le niveau d'ingestion des animaux est maximum. SHELLEMBERGER et KESLER (I96I) ont également trouvé une corrélation hautement significative entre la vitesse de transit digestif de la ration (mesurée sur le foin) et le niveau d'ingestion.

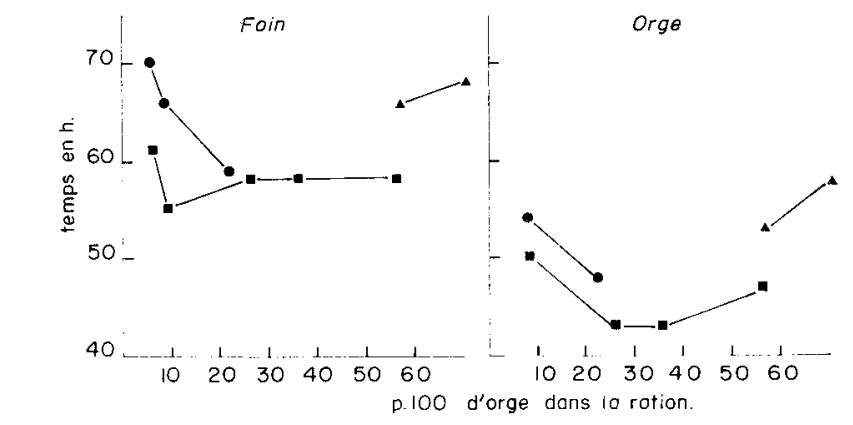

FIG. 2. - Temps de séjour moyen du toin el de l'orge dans le tube digestit

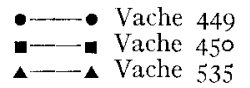

Le poids de contenu de rumen en matière fraîche et en matière sèche augmente avec le pourcentage d'orge jusqu'à un maximum qui a correspondu à 30 p. Ioo d'orge pour la Vache 450 . Il diminue ensuite de façon importante notamment pour les pourcentages d'orge supérieurs à 70 p. roo. Il présente ainsi une évolution parallèle à celle de la vitesse de transit digestif et des quantités ingérées.

L'activité fermentaire du rumen, mesurée par la quantité de gaz dégagée au cours des incubations de contenu de rumen, augmente jusqu'à 45 p. roo d'orge dans la ration puis semble ne plus augmenter ensuite. Cette augmentation est d'autant plus faible que le prélèvement de contenu est plus éloigné de la distribution (fig. 3). Pour le prélèvement effectué à I 5 heures, elle a même été nulle. 
L'activité cellulolytique du jus de rumen, mesurée par le pourcentage de disparition de papier-filtre placé dans des sachets de nylon plongés dans le rumen, diminue presque linéairement chez les vaches 449 et 450 de 7,6 points, pour une augmentation de Io points du pourcentage d'orge dans la ration entre 6 et $56 \mathrm{p}$. Ioo chez la Vache 450 . Malgré cette diminution, la digestibilité du foin jusqu'à 36 p. Ioo d'orge dans la ration semble supérieure à celle du foin seul, obtenue sur moutons (du moins avec le mode de calcul que nous avons employé).

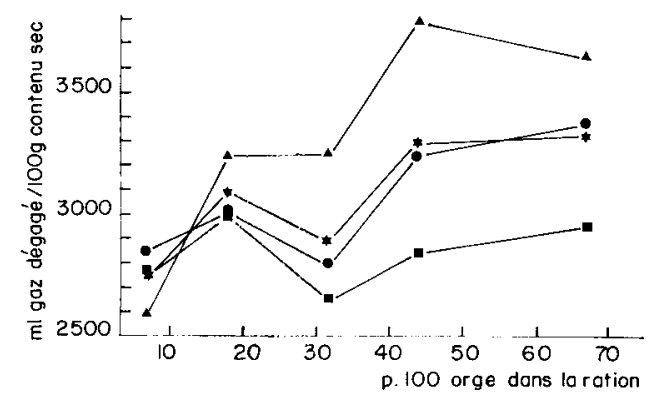

FIG. 3. - Incubations des contenus de rumen de la Vache 450
- Prélèvement effectué à 9 heures $\int$ Moyenne de

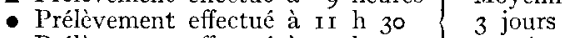
- Prélèvement effectué à I 5 heures consécutifs

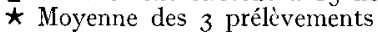

Le $\mathrm{pH}$ du jus de rumen prélevé par aspiration dans le milieu du sac ventral du rumen ne varie pas avec le pourcentage d'orge dans la ration. Il reste élevé (fig. 4) sans fluctuations importantes au cours de la journée malgré de fortes variations de la concentration du jus de rumen en $\mathrm{AGV}$, dues probablement en partie à notre méthode de prélèvement (irrégularité de débit de jus de rumen). La concentration en AGV semble avoir été maximum (I $9 \mathrm{~m} \mathrm{Mol} / 1$ ) avec $45 \mathrm{p}$. Ioo d'orge dans la ration et minimum (98 et $80 \mathrm{~m} \mathrm{Mol} / 1$ ) respectivement avec des proportions d'orge faibles (9 p. Ioo) et élevées (6o p. roo). La composition molaire reste très semblable, indépendante de la proportion d'orge, tout au moins pour l'acide acétique. Seule 1a proportion des acides butyriques et valérianiques a tendance à s'accroître au détriment de l'acide propionique.

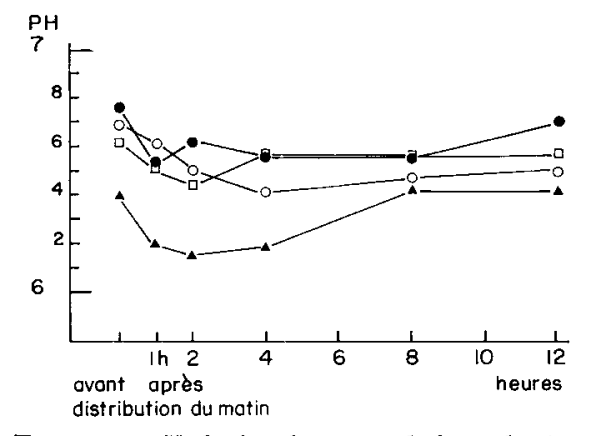

FIG. 4. $\rightarrow$ pH du jus de rumen de la vache 450

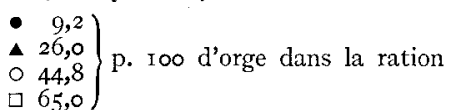


A l'inverse des quantités de gaz dégagées au cours des incubations et de la concentration du jus de rumen en $\mathrm{AGV}$, la stabilité du $\mathrm{pH}$ du jus de rumen et sa composition molaire en $\mathrm{AGV}$ suggèrent que l'activité fermentaire dans le rumen n'a pas beaucoup augmenté avec l'accroissement des quantités d'orge. La contradiction n'est peut-être qu'apparente. Bien que le pourcentage du temps total passé à ingérer et à ruminer ait diminué de $42 \mathrm{p}$. Ioo, il est possible que les substances tampons apportées par la salive aient suffi à neutraliser l'accroissement éventuel de la production des $\mathrm{AGV}$, maintenant ainsi le $\mathrm{pH}$ à un niveau élevé et le mélange des $\mathrm{AGV}$ dans sa composition initiale.

\title{
Régulation de l'ingestion
}

Le nombre d'animaux mis en expérience et les accidents limitent la portée de nos résultats. Néanmoins, il apparaît que les pourcentages d'orge dans la ration pour lesquels ont lieu la vitesse maximale d'ingestion et l'ingestion maximale de MS soient différents : la vache 450 ingère la quantité maximale de MS entre environ 36 et $45 \mathrm{p}$. Ioo d'orge dans la ration, mais n'atteint sa vitesse maximale d'ingestion (quantité de MS ingérée par unité de temps) que pour un pourcentage d'au moins $55 \mathrm{p}$. Ioo pendant la période 5). On peut donc penser qu'à partir de $45 \mathrm{p}$. Ioo d'orge dans la ration, des informations d'origine digestive ou métabolique empêchent l'animal d'ajuster son niveau d'ingestion à ses " goûts ".

Pendant la phase d'augmentation des quantités ingérées, la vitesse de dégradation des aliments dans le rumen et la digestibilité de la ration sont accrues. Il en est de même pour la vitesse de transit de l'orge et aussi, semble-t-il, celle du foin, malgré la réduction de l'activité cellulolytique du jus de rumen. L'animal peut donc ingérer davantage, l'augmentation de l'ingestion s'accompagnant d'un accroissement de la quantité de MS du contenu de rumen.

Pendant la phase de diminution des quantités ingérées, les vitesses de transit de l'orge et $\mathrm{du}$ foin ont tendance à diminuer et la vitesse de fermentation des aliments n'augmente plus. Or, la quantité de contenu de rumen diminue. La diminution de l'ingestion doit donc être recherchée dans des informations ayant pour origine non plus le rumen, mais les compartiments postérieurs, un changement du "gotit " de l'animal (du moins à partir du pourcentage d'orge pour lequel la vitesse d'ingestion diminue) et (ou) des informations d'origine métabolique. L'engraissement des animaux n'a pu que contribuer à la réduction de l'ingestion à la fin de l'expérience.

Reçu pour publication en nơ'embre rg68.

\author{
SUMMARY
}

EFFECT OF INCREASING AMOUNTS OF BARLEY ON FEEDING BEHAVIOUR

AND DIGESTION IN DAIRY COWS

Three dry cows with rumen fistulae were given nomal lucerne hay to appetite and increasing amounts of ground barley. Changes in feeding behaviour and digestion were studied (table i).

Intake of dry matter increased up to the point where barley represented 35 to 45 per cent of ration (fig. 1 ). 'This increase was accompanied by increases in the rate of passage of barley and hay 
through the digestive tract, the amount of rumen contents and digestibility of the ration (table 2). Rate of fermentation in the rumen also increased although cellulolytic activity of rumen fluid diminished.

With more than 35 to 45 per cent barley, intake of dry matter decreased. Rate of fermentation in the rumen did not increase any more and rate of passage of barley and hay tended to fall. Amount of rumen contents diminished. 'Thus the factors which limit intake can no longer originate in the rumen; they might originate in the posterior parts of the digestive tract or be of metabolic origin but they might also be caused by reduction in appetite of the cow since rate of intake decreased when 55 jer cent barley was given.

\section{RÉFÉRENCES BIBLIOGRAPHIQUES}

Gacsistes B., 1965. Méthode de prélèvement automatique de la phase liquide des contenus de rumen. Ann. Biol. anim. Bioch. Biophys., 5, 407-4I I.

Jotrnet M., Demarguidiy C., I967. Valeur alimentaire des foins condensés. II. Influence du broyage et de la mise en argrlomérés sur la diyrestion du foin de luzerne dans le rumen. . Inn. Zoolech. 16, 307-321.

KeSLER E. M., SPAHR S. L., 1964. Symposium : Effect of various levels of grain feeding. Physiological effects of hirh level concentrate fecding. J. Dairy Sci., 47, I $122-1128$.

Ruckeresci Y., 1963. Recherches sur la régulation centrale du comportement alimentaire chez les Ruminants. Thise Doct. Sci. Lyon.

Shem.enberger P. R., Kesler li. M., 1961. Rate of passage of feeds through the digestive tract of $/$ ols tein cows. J. Anim. Sci., 20, 410-+19.

Rook J. A. F., г 961 . Variations in the chemical composition of the milk of the cow. Part r. Dairy Sci. Abstr, 23, 25I-258.

VANIDENIELYET, F. A., 1964 . Rapid, accurate microanalysis of the lower fatty acids with particular reference to serial determination. Analyt. Chem., 36, 1930-1936. 